\title{
Incidencia de fracturas dentales reportadas en la Ciudad de Chihuahua durante la pandemia por COVID-19.
}

\author{
Incidence of dental fractures reported in Chihuahua city during COVID-19 pandemic.
}

\author{
Alfonso Espinosa Torres, * Julia Angélica Morales Ramos ${ }^{\ddagger}$
}

RESUMEN

Se presentan los casos de fracturas verticales sintomáticos que ameritaron la extracción de las órganos dentales afectados en un periodo de cinco meses durante la pandemia por COVID-19 en la ciudad de Chihuahua, México. Material y métodos: Se recolectaron 26 muestras para el estudio, proporcionadas por los cirujanos dentistas integrantes del Grupo de Egresados de la Facultad de Odontología de la Universidad Autónoma de Chihuahua. Resultados: Los resultados muestran diferencias a lo reportado previamente respecto al tipo de diente involucrado, sexo de los pacientes, restauraciones coronales y tratamientos endodóncicos previos. Conclusiones: Se puede suponer que las modificaciones en el estilo de vida de la población, sumadas a la angustia permanente, pueden ser factores que contribuyen a las fracturas dentales.

Palabras clave: Fractura vertical, dientes, COVID-19.
ABSTRACT

There are cases of symptomatic vertical fractures that warranted the extraction of affected teeth are presented, over a period of five months during the COVID-19 pandemic in Chihuahua City, Mexico. Material and methods: 26 samples were collected for the study, provided by the dentist who were members of the Graduate Group of the Faculty of Dentistry of the Autonomous University of Chihuahua. Results: The results show differences from what was previously reported regarding the type of tooth involved, sex of patients, coronal restorations and previous endodontic treatments. Conclusions: It can be assumed that changes in the lifestyle of the population, added to permanent distress, may be factors to preserve dental fractures.

Keywords: Vertical fracture, teeth, COVID-19.

\section{INTRODUCCIÓN}

$\mathrm{C}$ on motivo de la pandemia por COVID-19 (causado por virus SARS-CoV-2) las autoridades sanitarias limitaron durante un tiempo la atención odontológica sólo a pacientes cuyo motivo de consulta se consideraba tratamiento de emergencia, siempre y cuando no tuvieran síntomas respiratorios y temperatura dentro de rangos normales. ${ }^{1}$

Pese a lo anterior, la necesidad de tratamiento dental urgente disminuyó en 38 \% durante el brote COVID-19. Lo anterior indica que aún durante el estado de pandemia sigue existiendo necesidad de atención odontológica. ${ }^{2}$
Las fracturas dentales son un motivo de atención de urgencia dental por el dolor que provocan en el paciente. ${ }^{2}$

A las fracturas radiculares verticales (FRV) se asocian múltiples factores como postes, restauraciones intracoronales, condensación lateral de gutapercha $(84 \%)$, resorciones radiculares, contactos oclusales prematuros, bruxismo, bricomanía, ${ }^{3}$ traumatismos, ${ }^{4}$ iatrogenias ${ }^{5-7}$ y amelogénesis imperfecta. ${ }^{8}$

La fractura radicular vertical (FRV) es una lesión que se extiende longitudinalmente a lo largo de la raíz dental, comunicando conducto con periodonto. Esta fractura puede ser completa o incompleta y afectar esmalte, dentina, cemento y pulpa. ${ }^{9}$

\footnotetext{
* Egresado de la Universidad del Ejército y Fuerza Aérea Mexicanos, Profesor. ¥ Docente de la Facultad de Odontología.
}

Universidad Autónoma de Chihuahua (UACH).

Recibido: 13 de mayo de 2021. Aceptado: 18 de mayo de 2021.

Citar como: Espinosa TA, Morales RJA. Incidencia de fracturas dentales reportadas en la Ciudad de Chihuahua durante la pandemia por COVID-19. Rev ADM. 2021; 78 (3): 135-141. https://dx.doi.org/10.35366/100070 
La incidencia de FRV es mayor en premolares superiores con tratamiento previo de endodoncia $48.68 \%$ y aumenta en dientes restaurados con postes endodóncicos, probablemente debido a dos hipótesis: el diámetro mesiodistal más pequeño propicia la concentración de tensión en la raíz y la falta de una unión efectiva entre la raíz y poste aumenta el riesgo de FRV, por lo que se recomienda estar alerta cuando pacientes refieren dolor ligero a masticación y cumplen con las características mencionadas. ${ }^{9-11}$

Existen diferentes opiniones respecto la incidencia de FRV, un estudio determinó que son más comunes en premolares (82\%) que en molares (53\%) y típicamente en direcciones opuestas (no lineales), en dicho estudio la línea FRV se extendía oblicuamente de vestibular a palatino. ${ }^{12}$

Otros autores consideran que las fracturas dentarias constituyen las lesiones de origen traumático más comunes en la dentición permanente. ${ }^{13,14}$ La prevalencia varía de $26-76 \%$ de las lesiones dentales. ${ }^{15}$

Las fracturas dentarias se clasifican de acuerdo con Andreasen en infracciones del esmalte, fracturas no complicadas (esmalte o esmalte-dentina) y en fracturas complicadas que afectan esmalte, dentina y pulpa. ${ }^{15}$

Su prevalencia es alta, siendo los incisivos centrales superiores, los dientes más afectados en la arcada. Etiológicamente, se producen al golpear los dientes anteriores, de forma directa con objetos duros a alta velocidad. ${ }^{14}$

La clasificación de las fracturas coronarias se define de la siguiente forma:

1. Fracturas no complicadas de la corona:

a. Infracción de esmalte: cuando se produce una fractura incompleta del esmalte (grieta o fisura), sin pérdida de sustancia dental.

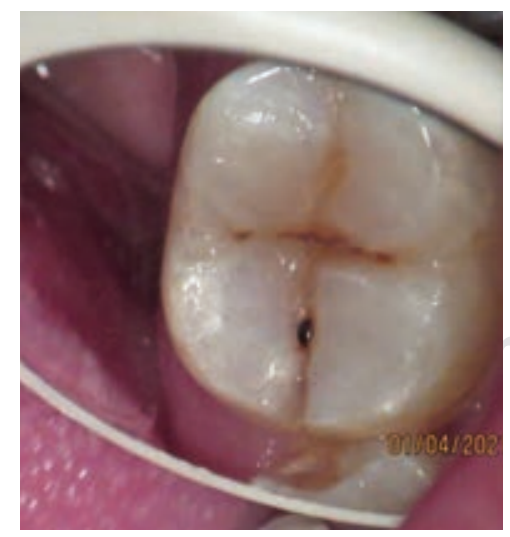

Figura 1: Microfotografía 10X de molar inferior con fractura en sentido M-D en molar inferior

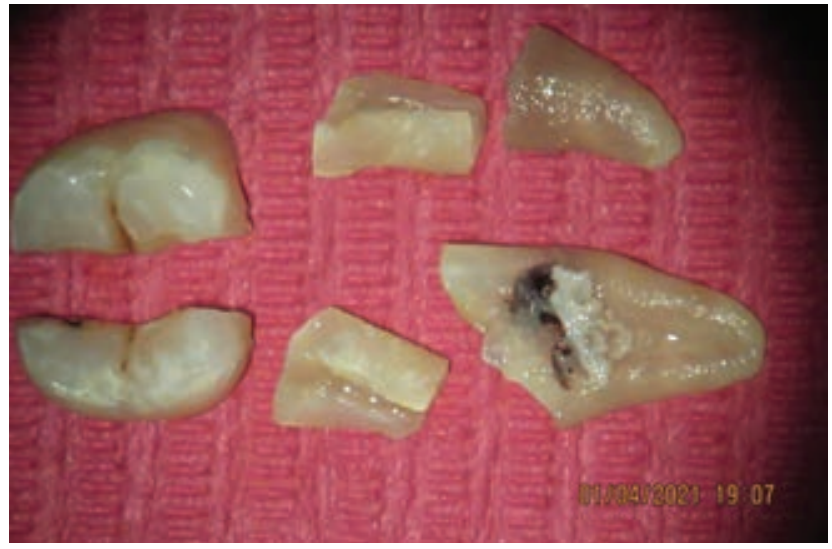

Figura 2: Micrografía 10X del molar inferior después de ser extraído.

Figura 3:

Radiografía digital de premolar superior después de ser extraído por presentar fisura intratable.

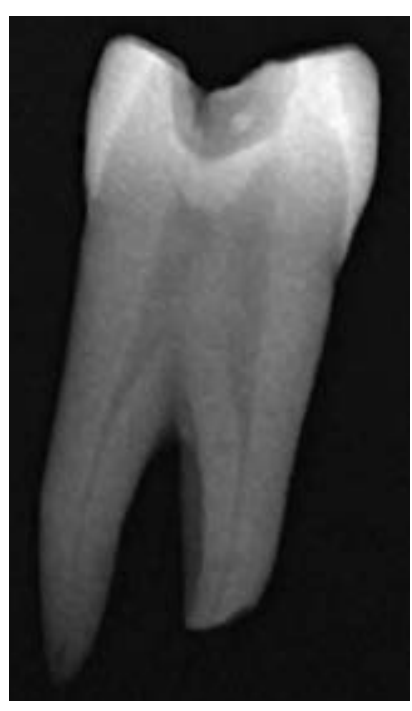

b. Fractura de esmalte: cuando se produce una fractura con pérdida de sustancia dental, pero localizada en el esmalte.

c. Fractura esmalte-dentina: cuando se produce una fractura con pérdida de sustancia dental que abarca al esmalte y a la dentina, pero sin compromiso pulpar.

2. Fracturas complicadas de la corona.

Fractura esmalte-dentina con compromiso pulpar

Afectan al esmalte, la dentina y además hay una exposición del tejido pulpar al medio bucal. Se evidencia por lo general un punto de hemorragia que corresponde a la pulpa expuesta, seguida de una respuesta inflamatoria superficial a causa de su contacto con productos de degradación y 


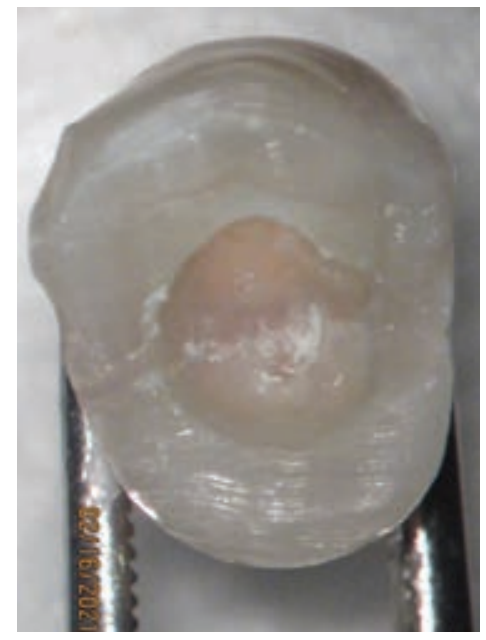

Figura 4:

Microfotografía 10X del premolar superior con fractura en sentido M-D que es poco visible.

bacterias. Posteriormente se produce coagulación y en días sucesivos pueden aparecer cambios degenerativos (abscesos o necrosis) o proliferativos (hiperplasia pulpar). ${ }^{14-18}$

Las fracturas coronales suelen producirse en los incisivos maxilares con una prevalencia de $96 \%$ con respecto al resto de los dientes. Dentro de este grupo, los incisivos centrales se ven afectados en aproximadamente $80 \%$ de los casos con respecto a los incisivos laterales. ${ }^{13}$

De acuerdo con lo descrito en la literatura, el género masculino es el más afectado en una proporción 3:1 con respecto al femenino y las edades más susceptibles oscilan entre los siete y 20 años. ${ }^{17}$

La amelogénesis imperfecta (AI) es una alteración que se presenta en el esmalte, básicamente en su estructura, en cuya formación intervienen tres proteínas básicas que son: enamelina, tuftelina y amelogenina. La tuftelina es la proteína más importante porque se encarga de iniciar el proceso de mineralización. Se describen tres tipos de Al: a) hipoplásico, b) hipomineralizado, y c) hipomaduro. Las personas que sufren de esta patología presentan alteraciones en el color del diente, y sensibilidad dentaria, y van a ser más susceptibles a caries dental y a fracturas. ${ }^{19-21}$

El bruxismo (BX) es considerado una parafunción oral-mandibular, se caracteriza por la presencia de movimientos músculo-mandibulares repetitivos sin propósitos funcionales aparentes, principalmente apretamiento y/o rechinamiento dental con gran acción nociva para el sistema estomatognático, ya que puede causar pérdida extensa de tejido dental por atrición (bruxofacetas) o abfracción, fracturas dentales, hipersensibilidad dental, dolor en la musculatura orofacial, disfunción masticatoria y articulación temporomandibular, siempre y cuando la intensidad de las fuerzas que se ejerzan logren superar la capacidad adaptativa de los mismos. La etiopatogenia y fisiopatología exacta del BX es aún desconocida, por lo que en la actualidad se considera de naturaleza multifactorial, regulado principalmente por el sistema nervioso central (SNC) e influido por factores periféricos. ${ }^{22,23}$

Los pacientes que presentan situaciones de ansiedad tienen más riesgo de sufrir un traumatismo dental. ${ }^{24}$

Se investigaron los niveles de catecolaminas urinarias en personas con historia previa de traumatismos dentales, observando que los que habían tenido una experiencia traumática, mostraban niveles significativamente altos de epinefrina con relación a los que no habían sufrido traumatismo. Para confirmar la hipótesis, los mismos autores realizaron estudios prospectivos con los mismos pacientes durante dos años, confirmando que cerca de $46 \%$ volvían a tener una lesión, y que los niveles de catecolaminas urinarias se mantenían altos. Estos datos avalan que el estado emocional es un prominente factor de riesgo en las lesiones dentofaciales. ${ }^{25,26}$

El presente estudio tiene por objeto analizar la incidencia de fracturas verticales en órganos dentales sufridas por pacientes durante una etapa de la pandemia de COVID-19 en la población del estado de Chihuahua, no relacionadas con traumatismos externos o accidentes.

\section{MATERIAL Y MÉTODOS}

Para el presente estudio se convocó a los integrantes del Grupo de Egresados de Odontología de la Universidad Autónoma de Chihuahua, conformado por 200 miembros, a reportar los casos de fisuras o fracturas dentales en pacientes sintomáticos que ameritaran extracción del órgano

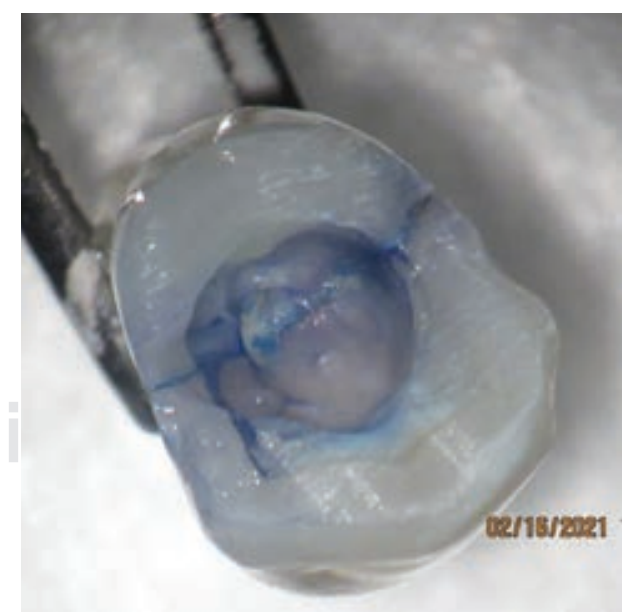

Figura 5: Microfotografía 10X de premolar superior con fractura en sentido M-D teñido con azul de metileno. 


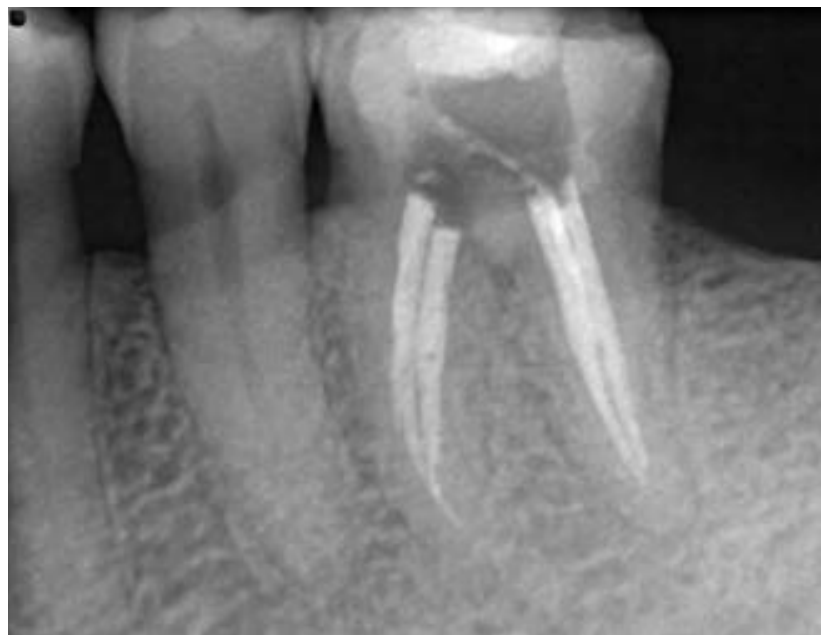

Figura 6: Radiografía periapical de molar inferior con fisura en sentido vestibulomesial previa a su extracción.

dental afectado, en el periodo del $1^{\circ}$ de julio de 2020 al 31 de marzo de 2021. No se consideraron en este estudio las fracturas ocasionadas por traumatismos externos.

Se seleccionaron únicamente aquellos casos en los que se pudo obtener evidencia clínica, fotográfica y radiográfica de la presencia de por lo menos una fisura vertical o fractura dental que provocara síntomas en el paciente y que ameritara la extracción del órgano dental por no ser tratable (Figuras 1 a 9).

Se recolectaron en total 26 órganos dentales, los cuales fueron fotografiados con microscopio dental para comprobar la presencia de las fisuras y/o fracturas, en algunos casos se realizó una tinción con azul de metileno para mejorar la visión de las lesiones.

Se agruparon en una tabla por sexo, edad, diente afectado y fecha de presentación, además se estableció si se tenía o no un tratamiento endodóncico en órgano dental afectado y en caso de tener tratamiento endodóncico, se registró si se les había colocado endoposte; también se anotó si el órgano dental estaba restaurado y de ser así con qué material; se estableció la dirección de la fisura o fractura en los diferentes planos y por último, se observó si el diente afectado tenía alguna lesión cariosa (Tabla 1).

\section{RESULTADOS}

Los resultados de la presente investigación mostraron lo siguiente: $38.4 \%$ de los casos correspondió a varones y $61.6 \%$ a mujeres, todos los casos se manifestaron en mayores de 40 años, $61.5 \%$ de los casos se presentaron en molares inferiores, $19.2 \%$ en molares superiores y $19.3 \%$ en premolares superiores (Figura 10).

Cuarenta y dos porciento de los casos tenían tratamiento de endodoncia previo y sólo a uno de ellos le habían colocado un endoposte metálico colado. Cincuenta y ocho porciento de los casos no tenía tratamiento de endodoncia (Figura 11).

Cincuenta y ocho porciento de los dientes afectados contaba con algún tipo de restauración coronal, siendo la más frecuente la restauración con resina con $46 \%$. Sólo una pieza contaba con restauración de corona metal porcelana, ésta era la misma pieza que contaba también con un endoposte.

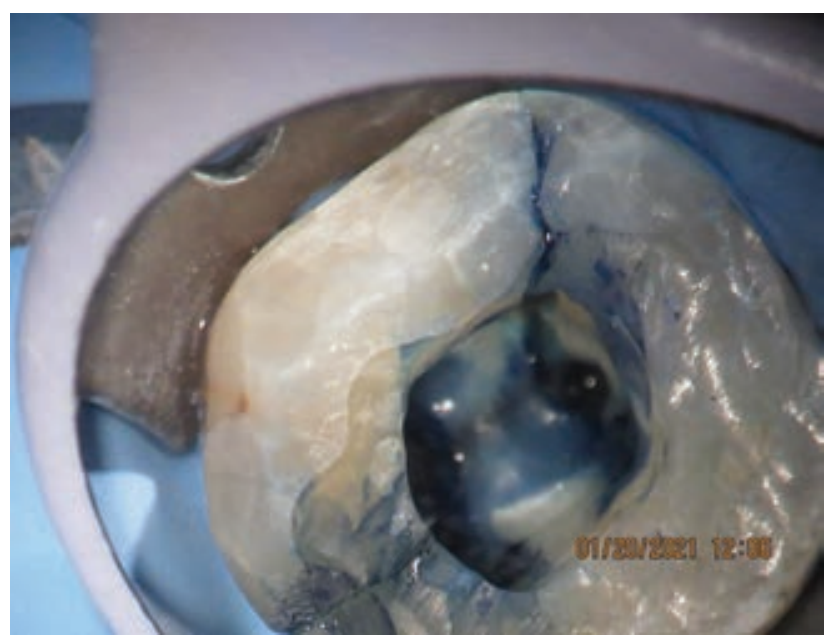

Figura 7: Microfotografía 10X del molar inferior con fractura en sentido V-M teñido con azul de metileno.

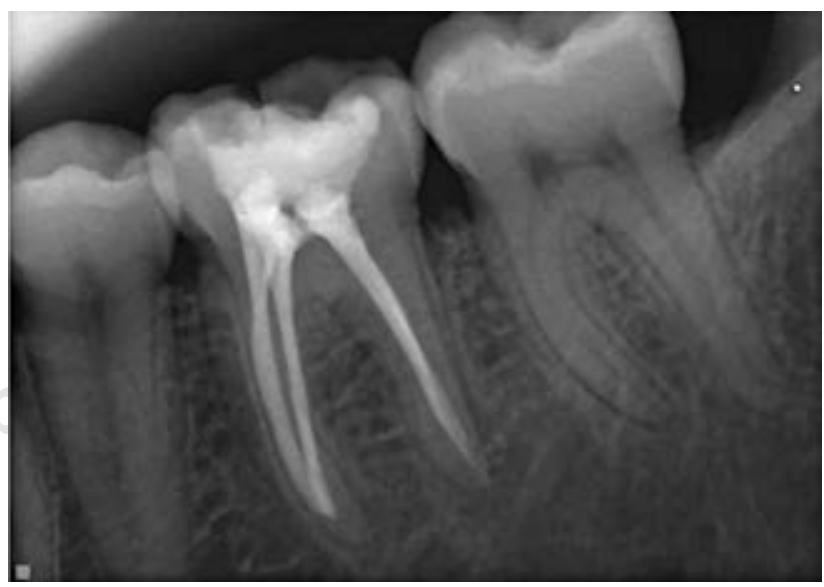

Figura 8: Radiografía periapical de molar inferior con tratamiento endodóncico y fisura en sentido mesiodistal previa a su extracción. 
Por otro lado, $88.5 \%$ de los casos no presentaba lesiones por caries visibles al momento de ser extraídas.

Se detectó también que seis de los órganos dentales no mostraban lesiones por caries ni tenían restauración alguna, es decir, que se trataba de dientes completos y sanos, esto equivalía a $15.6 \%$ de los casos.
De los 11 dientes que tenían tratamiento endodóncico previo, cuatro no presentaban restauración coronal, seis contaban sólo con restauración con base de resina y el restante, como ya se mencionó, tenía una restauración de metal-porcelana.

Respecto a la aparición de los casos, se reportaron tres en noviembre de 2020, seis en diciembre de 2020,

Tabla 1: Recolección de datos.

\begin{tabular}{|c|c|c|c|c|c|c|c|c|c|c|c|c|}
\hline \multirow[b]{2}{*}{ Muestra } & \multirow[b]{2}{*}{ Fecha } & \multirow[b]{2}{*}{ Sexo } & \multirow[b]{2}{*}{ Edad } & \multicolumn{2}{|c|}{$\begin{array}{l}\text { Tratamiento } \\
\text { endodóncico }\end{array}$} & \multicolumn{2}{|c|}{ Endoposte } & \multicolumn{2}{|c|}{ Restaurado } & \multicolumn{2}{|c|}{$\begin{array}{l}\text { Lesión por } \\
\text { caries }\end{array}$} & \multirow{2}{*}{$\begin{array}{l}\text { Fractura } \\
\text { Sentido }\end{array}$} \\
\hline & & & & Sí & No & Sí & No & Sí & No & Sí & No & \\
\hline 1 & 30-Diciembre-20 & $\mathrm{F}$ & 35 & & $\mathrm{x}$ & & & & & & $\mathrm{x}$ & M-D \\
\hline 2 & 08-Diciembre-20 & $\mathrm{F}$ & 45 & $\mathrm{x}$ & & & $\mathrm{x}$ & & $\mathrm{x}$ & $\mathrm{x}$ & & M-D \\
\hline 3 & 10-Febrero-21 & M & 34 & $\mathrm{x}$ & & $\mathrm{x}$ & & Corona & & & $\mathrm{x}$ & M-D \\
\hline 4 & 24-Febrero-21 & F & 46 & $\mathrm{x}$ & & & $\mathrm{x}$ & & $\mathrm{x}$ & & $\mathrm{x}$ & M-D \\
\hline 5 & 02-Diciembre-20 & $\mathrm{F}$ & 45 & & $\mathrm{x}$ & & $\mathrm{x}$ & & & & $\mathrm{x}$ & M-D \\
\hline 6 & 09-Febrero-21 & F & 48 & & $\mathrm{x}$ & & $\mathrm{x}$ & & $\mathrm{x}$ & & $\mathrm{x}$ & M-D \\
\hline 7 & 09-Febrero-21 & $\mathrm{F}$ & 51 & & $\mathrm{x}$ & & $\mathrm{x}$ & Resina & & & $\mathrm{x}$ & M-D \\
\hline 8 & 02-Febrero-21 & $\mathrm{F}$ & 46 & $\mathrm{x}$ & & & $\mathrm{x}$ & & $\mathrm{x}$ & & $\mathrm{x}$ & M-D \\
\hline 9 & 01-Diciembre-20 & F & 43 & $\mathrm{x}$ & & & $\mathrm{x}$ & & $\mathrm{x}$ & & $\mathrm{x}$ & M-D \\
\hline 10 & 21-Diciembre-20 & $\mathrm{F}$ & 56 & & $\mathrm{x}$ & & $\mathrm{x}$ & Resina & & $\mathrm{x}$ & & V-L \\
\hline 11 & 20-Enero-21 & M & 54 & & $\mathrm{x}$ & & $\mathrm{x}$ & Resina & & & $\mathrm{x}$ & M-D \\
\hline 12 & 04-Enero-21 & M & 48 & & $\mathrm{x}$ & & $\mathrm{x}$ & & $\mathrm{x}$ & & $\mathrm{x}$ & M-D \\
\hline 13 & 10-Noviembre-20 & M & 50 & & $\mathrm{x}$ & & & Resina & & $\mathrm{x}$ & & M-D \\
\hline 14 & 02-Febrero-21 & F & 43 & $\mathrm{x}$ & & & $\mathrm{x}$ & Resina & & & $\mathrm{x}$ & M-D \\
\hline 15 & 20-Marzo-21 & M & 43 & & $\mathrm{x}$ & & $\mathrm{x}$ & Resina & & & $\mathrm{x}$ & M-D \\
\hline 16 & 03-Febrero-21 & $\mathrm{F}$ & 39 & $\mathrm{x}$ & & & $\mathrm{x}$ & Temporal & & & $\mathrm{x}$ & V-L \\
\hline 17 & 03-Febrero-21 & F & 40 & & $\mathrm{x}$ & & $\mathrm{x}$ & Resina & & & $\mathrm{x}$ & M-D \\
\hline 18 & 13-Julio-20 & M & 40 & & $\mathrm{x}$ & & $\mathrm{x}$ & & $\mathrm{x}$ & & $\mathrm{x}$ & M-D \\
\hline 19 & 29-Enero-21 & $\mathrm{F}$ & 45 & & $\mathrm{x}$ & & $\mathrm{x}$ & & $\mathrm{x}$ & & $\mathrm{x}$ & M-D \\
\hline 20 & 20-Noviembre-20 & F & 51 & & $\mathrm{x}$ & & $\mathrm{x}$ & & $\mathrm{x}$ & & $\mathrm{x}$ & M-D \\
\hline 21 & 22-Febrero-21 & $\mathrm{F}$ & 39 & & $\mathrm{x}$ & & $\mathrm{x}$ & $\begin{array}{c}\text { Amalga- } \\
\text { ma }\end{array}$ & & & $\mathrm{x}$ & M-D \\
\hline 22 & 10-Febrero-21 & F & 48 & $\mathrm{x}$ & & & $\mathrm{x}$ & Resina & & & $\mathrm{x}$ & M-D \\
\hline 23 & 18-Febrero-21 & M & 45 & $\mathrm{x}$ & & & $\mathrm{x}$ & Resina & & & $\mathrm{x}$ & M-D \\
\hline 24 & 06-Enero-21 & $\mathrm{F}$ & 45 & & $\mathrm{x}$ & & $\mathrm{x}$ & Resina & & & $\mathrm{x}$ & M-D \\
\hline 25 & 25-Noviembre-20 & $\mathrm{F}$ & 48 & $\mathrm{x}$ & & & $\mathrm{x}$ & Resina & & & $\mathrm{x}$ & M-D \\
\hline 26 & 16-Diciembre-20 & $\mathrm{F}$ & 44 & $\mathrm{x}$ & & & $\mathrm{x}$ & Resina & & & $\mathrm{x}$ & M-D \\
\hline
\end{tabular}

$\mathrm{F}=$ Femenino, $\mathrm{M}=$ Masculino . 


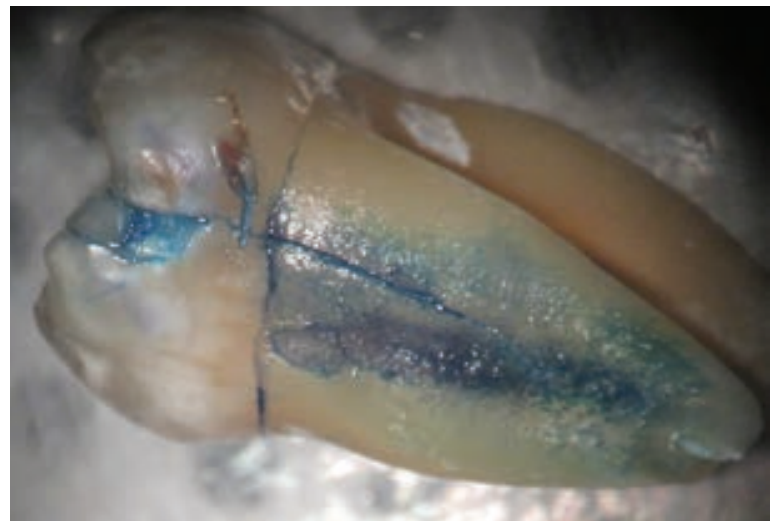

Figura 9: Microfotografía 10X de molar inferior con fisura en sentido M-D teñida con azul de metileno posterior a su extracción.

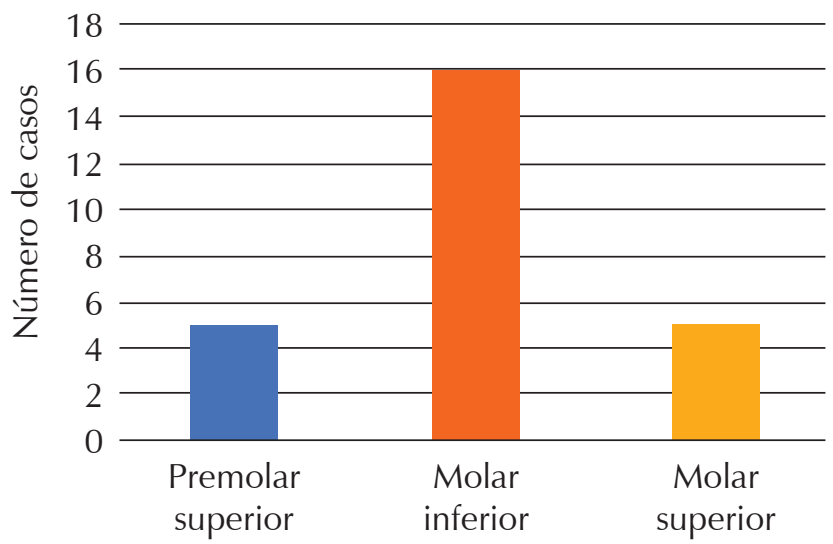

Fracturas por tipo de pieza dental

Figura 10: Distribución de las muestras por tipo de diente.

y en 2021 se registraron cuatro en enero, 11 en febrero y dos en marzo (Figura 12).

\section{DISCUSIÓN}

La hipersensibilidad dental puede ser uno de los primeros síntomas que muestran los pacientes ante la presencia de una fisura de esmalte. ${ }^{27}$ En este estudio la mayoría de los pacientes que no tenían tratamiento endodóncico sólo manifestaron dolor al aparecer la fractura dental.

Diferentes estudios mencionan que los premolares superiores son los más susceptibles a las fracturas verticales. ${ }^{9-11}$ En este trabajo, a diferencia de lo anterior, fueron los molares inferiores los que se presentaron en mayor número.
En este estudio la distribución por sexo no coincide con reportes similares, ${ }^{17}$ donde los hombres tienden a presentar con mayor frecuencia esta condición de fractura vertical. Las muestras de este trabajo reportaron $61 \%$ de casos en sexo femenino y $39 \%$ en sexo masculino.

Diversos autores también mencionan que los dientes con tratamiento endodóncico y una mala restauración coronal tienden a sufrir de fracturas y esta condición se incrementa en dientes con endopostes con un mal diseño; ${ }^{9-11}$ este estudio por el contrario, detectó una mayor proporción de casos en piezas dentales sin tratamiento endodóncico, tanto así que $60 \%$ de las muestras no tenían tratamiento de endodoncia, más aún, $23 \%$ de las muestras ni siquiera tenían lesiones por caries o restauraciones previas.

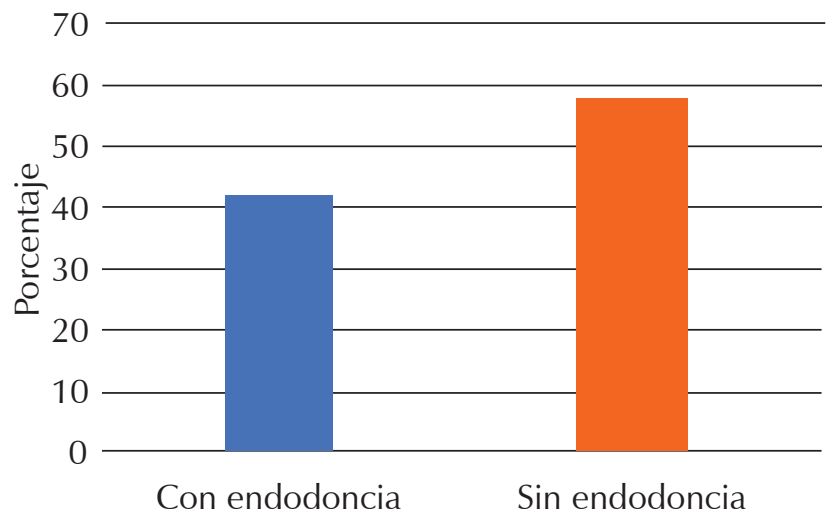

Figura 11: Distribución de muestras con y sin tratamiento de endodoncia previo.

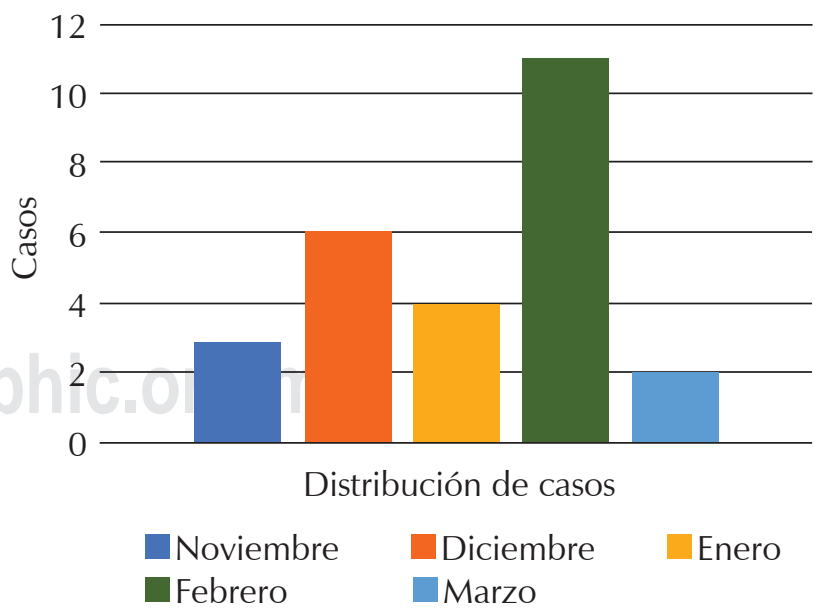

Figura 12: Distribución de casos por mes. 
Respecto a lo anterior, $57 \%$ de las muestras de este estudio tenían algún tipo de restauración coronal, siendo la más frecuente la restauración con resina tanto en piezas con tratamiento endodóncico previo y sin éste. Dentro de este grupo de muestras con restauraciones coronales, $80 \%$ correspondía a restauraciones elaboradas con resina.

\section{CONCLUSIONES}

La pandemia por COVID-19 ha modificado en todo el mundo las situaciones de vida, de trabajo y de salud de la población. En este estudio se observaron modificaciones en la incidencia y características de las fracturas verticales sintomáticas que se presentaron en órganos dentales y que ameritaron ser extraídos por no ser restaurables. Podemos suponer que las modificaciones en el estilo de vida de la población, sumadas a la angustia permanente que estas modificaciones provocan, pueden ser factores que contribuyeron a la presencia de este fenómeno. Para el diagnóstico de fisuras y/o fracturas la tinción con azul de metileno proporciona una mejor visión de éstas.

\section{AGRADECIMIENTOS}

A los cirujanos dentistas miembros del Grupo de Egresados de la Facultad de Odontología de la Universidad Autónoma de Chihuahua por su colaboración en el desarrollo del presente estudio.

\section{REFERENCIAS}

1. American Dental Association. ADA Interim Guidance or Management of Emergency and Urgent Dental Care. Accessible in: https://www.ada.org/ /media/CPS/Files/COVID/ADA_Int_ Guidance Mgmt Emerg-Urg Dental COVID19.pdf.

2. Guo H, Zhou Y, Liu X, Tan J. The impact of the COVID-19 epidemic on the utilization of emergency dental services. J Dent Sci. 2020; 15 (4): 564-567.

3. Yeh CJ. Fatigue root fracture: a spontaneous root fracture in nonendodontically treated teeth. Br Dent J. 1997; 182: 261-266.

4. Bender IB. Adult root fracture. J Am Dent Assoc. 1983; 107: 413-419.

5. Lam PP, Palamara JE, Messer HH. Fracture strength of tooth roots following canal preparation by hand and rotary instrumentation. J Endod. 2005; 31: 529-532.

6. Sathorn C, Palamara JE, Palamara D, Messer HH. Effect of root canal size and external root surface morphology on fracture susceptibility and pattern: a finite element analysis. J Endod. 2005; 31: 288-292.

7. Cohen S, Berman LH, Blanco L, Bakland L, Kim JS. A demographic analysis of vertical root fractures. J Endod. 2006; 32 (12): 1160-1163.

8. Sapir S, Shapira J. Clinical solutions for developmental defects of enamel and dentin in children. Pediatr Dent. 2007; 29 (4): 330-336.
9. Pitts DL, Natkin E. Diagnosis and treatment of vertical root fractures. J Endod. 1983; 9: 338-346.

10. Cohen S, Blanco L, Berman L. Vertical root fractures: clinical and radiographic diagnosis. J Am Dent Assoc. 2003; 134: 434-441.

11. Huang HM, Ou KL, Wang WN, Chiu WT, Lin CT, Lee SY. Dynamic finite element analysis of the human maxillary incisor under impact loading in various directions. J Endod. 2005; 31: 723-727.

12. Huang CC, Chang YC, Chuang MC, Lin HJ, Tsai YL, Chang SH et al. Analysis of the width of vertical root fracture in endodontically treated teeth by 2 micro-computed tomography systems. J Endod. 2014; 40: 698-702.

13. Goenka, P; Marwah, N; Dutta S. Biological approach for management of anterior tooth trauma: triple case report. J Indian Soc Pedod Prev Dent. 2011; 29 (2): 180-186.

14. García C, Mendoza A. Traumatología oral en odontopediatría. Diagnóstico y Tratamiento Integral. Madrid. Editorial: Ergon. 2003.

15. Andreasen JO, Andreasen FM, Andersson L. Texto y atlas a color de lesiones traumáticas a las estructuras dentales. Cuarta Edición. Oxford, Editorial AMOLCA, 2010.

16. Henostroza G. Adhesión en odontología restauradora. Editorial Ripano. Asociación Latinoamericana de Operatoria Dental y Biomateriales. 2a . ed. Madrid. 2010.

17. Stefanello A, González P, Prates R. Odontología restauradora y estética. Editorial Amolca. Brasil. 2005.

18. Boj JR, Catalá M, García C, Mendoza A. Odontopediatría. Barcelona. Editorial: Masson. 2005.

19. Witkop CJ Jr. Amelogenesis imperfecta, dentinogenesis imperfecta and dentin dysplasia revisited: problems in classification. J Oral Pathol. 1988; 17 (9): 547-553.

20. Martín-González J, Sánchez-Domínguez B, Tarilonte-Delgado M, Castellanos-Cosano L, Llamas-Carreras J, López-Frías F et al. Anomalías y displasias dentarias de origen genético-hereditario. Avances en Odontoestomatología. 2012; 28 (6): 287-301.

21. Ceballos O DM, Espinal GE, Jones M. Anomalías en el desarrollo y formación dental: odontodisplasia. Int J Odontostomat. 2015; 9 (1): 129-136.

22. De La Hoz-Aizpurua JL, Winocur E. Bruxismo del sueño: revisión actualizada de conceptos y utilidad de la toxina botulínica en su tratamiento. Rev Soc Esp Dolor. 2014; 21 (2): 4-13.

23. Castellanos SJ. Bruxismo. Nociones y conceptos. Rev ADM. 2015; 72 (2): 63-69.

24. Manfredini D, Restrepo C, Diaz-Serrano K, Winocur E, Lobbezoo F. Prevalence of sleep bruxism in children: a systematic review of the literature. J Oral Rehabil. 2013; 40 (8): 631-642.

25. Lange M. The bruxism status examination protocol. Journal of Craniomandibular Function. 2017; 9 (1): 57-69.

26. Alrusayes AA, Assal NA, Althobity AM, Alfraih YK, Alfraih MI et al. Dentin hypersensitivity: a review of its treatment. Annals of International Medical and Dental Research. 2021; 7 (3): 8-15.

Conflicto de intereses: Los autores manifiestan no tener conflicto de intereses.

Correspondencia:

Alfonso Espinosa Torres

E-mail: alfonso.endo@hotmail.com 